\title{
Treinamento de Sobrecarga Muscular Não Afeta o Diâmetro das Principais Veias dos Membros Inferiores em Mulheres Adultas com Insuficiência Venosa
}

\author{
Strength Training Does not Affect the Diamater \\ of the Main Veins of Lower Limbs in Adult Women \\ With Mild to Moderate Chronic Venous Insufficiency
}

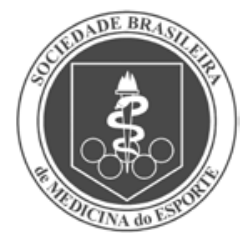

Artigo Original

\begin{abstract}
Gigliola Cibele Cunha da Silva² Rômulo José Dantas Medeiros',2,3 Leonardo dos Santos Oliveira2,3 Adenilson Targino de Araújo Júnior2,3 Rodrigo Ramalho Aniceto² Maria do Socorro Cirilo de Sousa ${ }^{1,2}$ Rodolfo Augusto Athayde Neto ${ }^{4}$

1. Departamento de Educação Física (DEF) - Universidade Federal da Paraíba (UFPB) - João Pessoa, PB - Brasil.

2. Grupo de Pesquisa em

Cineantropometria, Atividade Física e Saúde, Desenvolvimento e Desempenho Humano - GPCASD/CNPq. 3. Programa Associado de PósGraduação em Educação Física UFPB/ UPE - João Pessoa, PB e Recife, PE - Brasil.

4. Departamento de Cirurgia - UFPB João Pessoa, PB - Brasil.
\end{abstract}

Endereço para correspondência: Rômulo José Dantas Medeiros, Cidade Universitária - Campus I - 58059-900 - João Pessoa, PB - Centro de Ciências da Saúde (CCS) - Departamento de Educação Física (DEF).

E-mail: romuloaquazul@yahoo.com.br

\begin{abstract}
RESUMO
O exercício físico pode promover benefícios na funcionalidade do sistema venoso. Contudo, tratandose de exercício com sobrecargas musculares, observa-se contraindicações quanto à sua prática diante da possibilidade da mesma estar relacionada ao agravamento das disfunções venosas. Objetivo: Analisar os efeitos da prática do exercício com sobrecargas musculares sobre o diâmetro venoso de mulheres fisicamente inativas portadoras de insuficiência venosa crônica de membros inferiores. Métodos: Vinte e duas mulheres com idades entre 21 e 58 anos (34,27 \pm 12 anos) foram divididas aleatoriamente em dois grupos: experimental $(n=12)$ e controle $(n=10)$. O treinamento com cargas foi realizado por 16 semanas, e o diâmetro venoso foi medido nas veias safenas magna (em nível da coxa e perna) e parva no membro inferior esquerdo por meio de ecodoppler colorido. Utilizou-se a ANOVA por dois fatores para avaliar as possíveis modificações dos diâmetros entre os grupos e ao longo do tempo $(p<0,05)$. Resultados: Não foram encontradas alterações significativas nos diâmetros das veias safenas parva (porções superior, média e inferior: $p=0,80,0,32$ e 0,20, respectivamente), magna em nível da perna ( $p=0,17,0,74$ e 0,96) e magna em nível da coxa ( $p=0,57,0,67$ e 0,52). Conclusão: A prática do exercício com sobrecargas musculares pode ser considerada um meio de intervenção ou tratamento, uma vez que não promoveu alterações no diâmetro venoso de mulheres que apresentaram insuficiência venosa crônica nos membros inferiores.
\end{abstract}

Palavras-chave: exercício resistido, veias, extremidade inferior.

\section{ABSTRACT}

Physical exercise can promote benefits in the functionality of the venous system. However, in the strength training context, contraindication concerning its practice is observed, since it can be related to worsening of venous dysfunctions. Objective: To analyze the effects of resistance exercises practice on venous diameter of physical inactivity women with chronic lower limb venous insufficiency. Methods: Twenty-two women with age between 21 and 58 years (34.27 \pm 12 years) were randomized divided in two groups: experimental $(n=$ $12)$ and control $(n=10)$. The resistance training was carried out over a period of 16 weeks and the venous diameter measurements were determined at the left lower limb Great and Small saphenous veins by a color Eco- Doppler. Two-way ANOVA was used to determine the modifications in veins diameter between groups and training moments (pre and post) ( $p<0.05$ ). Results: Statistically significant alterations were not found in smaller saphenous vein diameter (upper, medium and lower points: $p=0.80 ; 0.32$ and 0.20 , respectively), great saphenous vein on leg level $(p=0.17 ; 0.74$ and 0.96$)$ and great saphenous vein on thigh level $(p=$ $0.57 ; 0.67$ and 0.52 ). Conclusion: Resistance exercise practice can be considered a treatment or intervention since it does not promote changes on the venous diameter of the venous system of women with chronic lower limb venous insufficiency.

Keywords: resistance exercise, vein, lower extremity. 


\section{INTRODUÇÃO}

No decorrer da última década, estudos demonstraram que a prática do exercício físico, em especial daquele realizado por meio do deslocamento de cargas, é capaz de promover inúmeras adaptações no corpo humano. Assim, evidencia-se o aperfeiçoamento de capacidades físicas inerentes à função do sistema musculoesquelético ${ }^{(1)}$ e alterações estruturais indicativas de aumentos de massa muscular ${ }^{(2)}$, densidade óssea ${ }^{(3)}$ e resistência do tecido conjuntivo(4).

A literatura reporta claramente como o exercício com cargas é capaz de produzir efeitos benéficos sobre o sistema neuromuscular ${ }^{(1)}$. Todavia, alterações cardiovasculares, principalmente no que se refere à funcionalidade do sistema venoso, ainda não são amplamente investigadas e tampouco completamente compreendidas. Quanto a esta questão, maior destaque tem sido destinado à análise dos efeitos do treinamento aeróbio sobre os componentes vasculares ${ }^{(5)}$.

$\mathrm{Na}$ atualidade, coloca-se em evidência que as doenças venosas, das quais fazem parte as varizes e a insuficiência venosa crônica (IVC), estão entre as disfunções que mais se encontram presentes como causa de morbidade na população de grande parte dos países ocidentais ${ }^{(6)}$. Diante desse fato, pesquisas são desenvolvidas com o objetivo de identificar tanto os fatores etiológicos das doenças venosas como os elementos ambientais capazes de acentuar ou diminuir sua gravidade.

Lima et al.(7) afirmam que a IVC é uma doença que além de acometer o âmbito pessoal do seu portador, causando dores, perdas de mobilidade funcional e piora da qualidade de vida, também provoca danos à esfera governamental. Abenhaim e Kurz ${ }^{(8)}$ destacam que a alta incidência de casos de IVC é acompanhada por elevados custos de tratamento. Nos Estados Unidos, por exemplo, esse custo é estimado em 1,9 a 2,5 bilhões de dólares por ano. No Brasil, por sua vez, a importância socioeconômica da IVC passou a ser considerada pelo governo apenas a partir da década de 1980, acarretando um crescente interesse no que se refere ao conhecimento científico e clínico das questões relacionadas a essa doença ${ }^{(9)}$.

No tocante aos fatores ambientais expressos pelas ações de intervenção, percebe-se que além dos procedimentos farmacológicos ${ }^{(10)}$, cirúrgicos $^{(11)}$ e alternativos (compressão elástica) ${ }^{(12)}$, atualmente o exercício físico é considerado uma medida efetiva de prevenção e tratamento da IVC, tendo a prática da caminhada um ponto de destaque nos estudos que investigam essa temática ${ }^{(13)}$. No âmbito dos exercícios neuromusculares, apenas o treinamento da musculatura da panturrilha é colocado em evidência como uma atividade capaz de diminuir o refluxo sanguíneo, aprimorar a competência das veias e consequentemente causar redução dos desconfortos e malefícios promovidos por tal disfunção(14).

Contudo, faz-se necessário destacar que o exercício com sobrecargas musculares desenvolvido para o treinamento de todos os principais grupos musculares do corpo, encontra-se bastante disseminado no cotidiano de praticantes de atividade física, sendo estes portadores ou não de IVC. Em se tratando dessa última população, observa-se um senso comum presente tanto nos centros de treinamento como nos discursos de profissionais das áreas da saúde, contraindicando a prática desse tipo de atividade por atribuir à mesma a acentuação do diâmetro venoso e o posterior agravamento do estado da doença. Ao se buscar dados na literatura capazes de corroborar ou contrapor tal senso comum, lacunas são identificadas não permitindo respostas conclusivas.

Diante desta perspectiva, o desenvolvimento do presente trabalho é norteado pela seguinte questão: a prática do exercício com sobrecargas musculares desenvolvido para o treinamento dos principais grupos musculares dos membros inferiores aumenta o diâmetro venoso? Frente ao exposto e sabendo-se que o nível de gravidade da IVC é acentuado quando o diâmetro venoso aumenta ${ }^{(15,16)}$, essa investigação tem como objetivo analisar os efeitos do exercício com cargas sobre o diâmetro venoso de mulheres fisicamente inativas portadoras de insuficiência venosa crônica de membros inferiores.

\section{MÉTODOS}

\section{Amostra}

A amostra foi constituída por 22 mulheres fisicamente inativas, com idades entre 21 e 58 anos (34,27 \pm 12 anos), subdivididas de forma aleatória em dois grupos: Experimental (GE) ( $n=12)$ e Controle (GC) ( $n=10)$. Para seleção da amostra foram distribuídos e fixados panfletos com a divulgação dos propósitos do estudo em ambientes da Universidade Federal da Paraíba (UFPB) e do Hospital Universitário $(H U)$. Para a randomização dos sujeitos nos grupos, utilizou-se a ferramenta online Research Randomizer da Social Psychology Network (www.randomizer.org). Aquelas que atendiam aos critérios elencados foram selecionadas para participar do estudo. As características individuais dos grupos GE e GC são apresentadas na tabela 1.

Como critérios de inclusão amostral foram consideradas para o estudo mulheres que apresentavam IVC nas classes de um a três, diagnosticada clinicamente por um médico angiologista (R.A.A.N.) segundo a classificação da CEAP (Clinical signs; Etiology; Anatomic distribution; Pathophysiology)(17), e que se encontravam sem realizar exercício físico regular por um período mínimo de seis meses. Foram retiradas do estudo aquelas que não apresentassem $75 \%$ de frequência ao programa de treinamento ${ }^{(18)}$.

Este estudo foi aprovado pelo Comitê de Ética em Pesquisa do Centro de ciências da Saúde da Universidade Federal da Paraíba sob o protocolo no 90/06/07, e as participantes assinaram um termo de consentimento livre e esclarecido, o qual continha informações sobre a natureza voluntária da amostra, objetivos do estudo, sigilo dos dados, entre outros aspectos éticos.

Tabela 1. Características antropométricas dos grupos experimental $(n=12)$ e controle $(n=10)$.

\begin{tabular}{l|c|c|c|c}
\hline \multicolumn{5}{c}{ Características da amostra } \\
\hline Variável & \multicolumn{2}{|c|}{ Grupo experimental } & \multicolumn{2}{c}{ Grupo controle } \\
\hline & Média \pm DPC & Mín.d - Máx.e & Média \pm DP & Mín. - Máx. \\
\hline Idade (anos) & $32,08 \pm 10,02$ & $21-56$ & $36,90 \pm 14,11$ & $22-58$ \\
\hline MC (kg)a & $59,37 \pm 8,66$ & $44,5-75$ & $53,93 \pm 9,96$ & $39,9-76,5$ \\
\hline Estatura $(\mathrm{m})$ & $1,59 \pm 0,04$ & $1,54-1,68$ & $1,56 \pm 0,10$ & $1,41-1,71$ \\
\hline IMC $\left(\mathrm{kg} / \mathrm{m}^{2}\right) \mathrm{b}$ & $23,23 \pm 2,78$ & $18,76 \pm 28,23$ & $21,92 \pm 3,74$ & $17,04-29,51$ \\
\hline
\end{tabular}

a: massa corporal; b: índice de massa corporal; c: desvio padrão; d: mínimo; e: máximo.

\section{Medidas antropométricas}

As medidas antropométricas foram realizadas apenas antes da intervenção, com o objetivo de caracterizar a amostra, tendo-se todas as medidas tomadas no Laboratório de Cineantropometria da UFPB, em horários padronizados para ambos os grupos (09h00min a 10h00min), dois dias antes do início do programa de exercícios. A massa corporal (MC) (kg) foi quantificada em uma balança antropométrica (Filizola, Brasil) com precisão de $100 \mathrm{~g}$ e capacidade máxima de $150 \mathrm{~kg}$ e a estatura (m) em um estadiômetro (Cardiomed, Brasil) com precisão de $1 \mathrm{~mm}$.

\section{Medidas do diâmetro venoso}

Todas as medidas do diâmetro venoso foram realizadas no membro inferior esquerdo, antes e após a intervenção em horários padronizados (07h00min a 08h30min) pelo angiologista, no qual fez uso de um ecodoppler colorido (Duplex Scan, Shimadzu 2200, Japão) com 
transdutor linear multifrequencial de 5-10MHz. O diâmetro venoso foi determinado nas veias safenas magna (VSM), nas porções superior, média e inferior da coxa (VSMC) e perna (VSMP), e parva (VSP), nas porções inferior, média e superior da perna. Para a realização dos exames, as voluntárias adotaram a posição de Trandelenburg reverso de $45^{\circ}$ de inclinação(19).

As medidas da coxa foram determinadas: i) na região inguinal a 2cm de distância da junção safenofemoral; ii) próxima à articulação do joelho a $3 \mathrm{~cm}$ do bordo superior da patela; e iii) em um ponto medial equidistante em relação às medidas das porções inferior e superior. Já as medidas da perna foram mensuradas: i) na região da panturriIha em uma altura equivalente a $3 \mathrm{~cm}$ de distância do bordo inferior da patela; ii) na região dorsomedial do pé no nível da articulação do tornozelo; e iii) em um ponto medial equidistante em relação às medidas verificadas na perna ${ }^{(20)}$. O médico não teve conhecimento de quais eram as mulheres que faziam parte dos grupos GE e GC, caracterizandose como um modelo cego de avaliação.

\section{Determinação da carga máxima (1-RM)}

O valor de 1-RM foi determinado segundo o modelo matemático de estimação de carga máxima proposto por Brzycki(21). Primeiramente, as voluntárias do GE foram submetidas a série de aquecimento de seis a 10 repetições (rep) com aproximadamente 50\% da carga estabelecida para o teste. Após um período de repouso de cinco minutos, o teste foi iniciado; tenta-se executar o máximo de repetições possíveis até que se configurasse uma incapacidade funcional de vencer a resistência oferecida. Se o número de repetições máximas (RM) realizadas fosse maior que 10 e inferior a sete, um ajuste de carga era efetuado e outra série executada depois de passados três a cinco minutos de descanso. Segundo Whisenant et al. ${ }^{(22)}$, a margem de sete a 10 RM deve ser utilizada devido ao fato de este número de repetições ser considerado o mais adequado para estimar os valores de 1-RM a partir de testes submáximos de força.

\section{Protocolo experimental}

O treinamento durou 16 semanas, tendo-se suas atividades implantadas na academia de ginástica do Departamento de Educação Física da UFPB, em horários padronizados (11 h30min a 13h30min). No período que abrangeu o programa, as componentes do GE compareceram três vezes por semana no local selecionado, e as sessões de exercício foram compostas por cinco exercícios para membros inferiores, sendo eles cadeira extensora, mesa flexora, leg press horizontal, panturrilha no leg press horizontal e panturrilha sentado (sentadilha).

Durante a intervenção, houve inicialmente uma fase de adaptação e outra específica. A primeira teve duração de quatro semanas, tempo necessário à adaptação neura|(1), e a específica de 12 semanas, sendo distintas apenas em relação à carga aplicada, que foi acrescida de 2-10\% quando os indivíduos conseguiam realizar uma ou mais repetições acima da quantidade prescrita(23).

Cada exercício envolveu a execução de três séries de seis-oito repetições e intervalos de 2-3min, com aquecimento de 10-15 min, durante, no total, cerca de 30-50min. Orientou-se a realização dos movimentos na fase concêntrica entre um-dois segundos e na fase excêntrica, de dois-quatro segundos, e as cargas utilizadas equivaleram-se a 70-85\% de uma repetição máxima (1-RM) $)^{(23)}$.

\section{Dados e análise estatística}

Os dados foram reportados na forma de valores médios e desvio padrão. Verificou-se a normalidade dos dados de interesse (teste de Shapiro Wilk) e a homogeneidade das medidas de diâmetro no momento pré-treinamento (teste de Levene). $O$ teste $t$ foi empregado para verificar diferenças entre os grupos para as variáveis antropométricas antes da intervenção. Em seguida, utilizou-se a análise de variância (ANOVA) por dois fatores para avaliar as possíveis modificações dos diâmetros das veias safenas magna e parva entre os grupos (GC e GE) e ao longo do tempo (pré e pós-treinamento). A análise foi executada pelo pacote SPSS 16.0 (SPSS Inc.,Chicago, IL, EUA), em que as diferenças foram consideradas significativas quando $p<0,05$.

\section{RESULTADOS}

Não foram verificadas diferenças significativas entre os grupos para as variáveis antropométricas antes da intervenção $(p>0,05)$ (tabela 1). Nas tabelas 2, 3 e 4 são apresentados os valores médios e desvio padrão dos diâmetros das veias magna, em nível da perna e coxa, e parva medidos antes e após a realização do treinamento. Não se observaram diferenças significativas $(p>0,05)$ nos momentos pré e pós-treinamento para ambos os grupos, assim como não foram identificados efeitos significantes do experimento em nenhuma das porções do diâmetro avaliadas.

Tabela 2. Diâmetros da veia safena magna em nível da perna nos momentos pré e pós-treinamento.

\begin{tabular}{|c|c|c|c|c|c|}
\hline Variável / teste & GEa $(n=10)$ & $\mathrm{GCb}(\mathrm{n}=10)$ & Efeitos & $F$ & $\mathbf{P}$ \\
\hline VSMPC Superior & & & ANOVA & & \\
\hline Pré-treinamento & $2,79 \pm 0,74$ & $2,89 \pm 0,61$ & Grupo & 0,16 & 0,69 \\
\hline Pós-treinamento & $2,59 \pm 0,71$ & $2,72 \pm 0,68$ & Treinamento & 3,57 & 0,07 \\
\hline$\Delta \%$ & $-7,72$ & $-2,94$ & $\begin{array}{c}\text { Grupo } \\
\text { treinamento }\end{array}$ & 0,23 & 0,88 \\
\hline VSMP Médio & & & ANOVA & & \\
\hline Pré-treinamento & $2,31 \pm 0,51$ & $2,44 \pm 0,59$ & Grupo & 0,16 & 0,69 \\
\hline Pós-treinamento & $2,26 \pm 0,53$ & $2,31 \pm 0,64$ & Treinamento & 0,68 & 0,41 \\
\hline$\Delta \%$ & $-2,21$ & $-5,62$ & $\begin{array}{c}\text { Grupo } \\
\text { treinamento }\end{array}$ & 0,1 & 0,74 \\
\hline VSMP Inferior & & & ANOVA & & \\
\hline Pré-treinamento & $2,45 \pm 0,48$ & $2,70 \pm 0,65$ & Grupo & 0,24 & 0,62 \\
\hline Pós-treinamento & $2,46 \pm 0,81$ & $2,47 \pm 0,65$ & Treinamento & 0,78 & 0,38 \\
\hline$\Delta \%$ & 0,4 & $-9,31$ & $\begin{array}{c}\text { Grupo } \\
\text { treinamento }\end{array}$ & 0,9 & 0,35 \\
\hline
\end{tabular}

Tabela 3. Diâmetros da veia safena magna em nível da coxa (média \pm desvio padrão) nos momentos pré e pós-treinamento.

\begin{tabular}{|c|c|c|c|c|c|}
\hline Variável / teste & GEa $(n=10)$ & $\mathrm{GCb}(\mathrm{n}=10)$ & Efeitos & $F$ & $\mathbf{P}$ \\
\hline VSMPC Superior & & & ANOVA & & \\
\hline Pré-treinamento & $2,79 \pm 0,74$ & $2,89 \pm 0,61$ & Grupo & 0,16 & 0,69 \\
\hline Pós-treinamento & $2,59 \pm 0,71$ & $2,72 \pm 0,68$ & Treinamento & 3,57 & 0,07 \\
\hline$\Delta \%$ & $-7,72$ & $-2,94$ & $\begin{array}{c}\text { Grupo } \\
\text { treinamento }\end{array}$ & 0,23 & 0,88 \\
\hline VSMP Médio & & & ANOVA & & \\
\hline Pré-treinamento & $2,31 \pm 0,51$ & $2,44 \pm 0,59$ & Grupo & 0,16 & 0,69 \\
\hline Pós-treinamento & $2,26 \pm 0,53$ & $2,31 \pm 0,64$ & Treinamento & 0,68 & 0,41 \\
\hline$\Delta \%$ & $-2,21$ & $-5,62$ & $\begin{array}{c}\text { Grupo } \\
\text { treinamento }\end{array}$ & 0,1 & 0,74 \\
\hline VSMP Inferior & & & ANOVA & & \\
\hline Pré-treinamento & $2,45 \pm 0,48$ & $2,70 \pm 0,65$ & Grupo & 0,24 & 0,62 \\
\hline Pós-treinamento & $2,46 \pm 0,81$ & $2,47 \pm 0,65$ & Treinamento & 0,78 & 0,38 \\
\hline$\Delta \%$ & 0,4 & $-9,31$ & $\begin{array}{c}\text { Grupo } \\
\text { treinamento }\end{array}$ & 0,9 & 0,35 \\
\hline
\end{tabular}


Tabela 4. Diâmetros da veia safena parva nos momentos pré e pós-treinamento.

\begin{tabular}{|c|c|c|c|c|c|}
\hline Variável / teste & GEa $(n=10)$ & $\mathrm{GCb}(\mathrm{n}=10)$ & Efeitos & $F$ & $\mathbf{P}$ \\
\hline VSMPC Superior & & & ANOVA & & \\
\hline Pré-treinamento & $2,79 \pm 0,74$ & $2,89 \pm 0,61$ & Grupo & 0,16 & 0,69 \\
\hline Pós-treinamento & $2,59 \pm 0,71$ & $2,72 \pm 0,68$ & Treinamento & 3,57 & 0,07 \\
\hline$\Delta \%$ & $-7,72$ & $-2,94$ & $\begin{array}{l}\text { Grupo } \\
\text { treinamento }\end{array}$ & 0,23 & 0,88 \\
\hline VSMP Médio & & & ANOVA & & \\
\hline Pré-treinamento & $2,31 \pm 0,51$ & $2,44 \pm 0,59$ & Grupo & 0,16 & 0,69 \\
\hline Pós-treinamento & $2,26 \pm 0,53$ & $2,31 \pm 0,64$ & Treinamento & 0,68 & 0,41 \\
\hline$\Delta \%$ & $-2,21$ & $-5,62$ & $\begin{array}{l}\text { Grupo } \\
\text { treinamento }\end{array}$ & 0,1 & 0,74 \\
\hline VSMP Inferior & & & ANOVA & & \\
\hline Pré-treinamento & $2,45 \pm 0,48$ & $2,70 \pm 0,65$ & Grupo & 0,24 & 0,62 \\
\hline Pós-treinamento & $2,46 \pm 0,81$ & $2,47 \pm 0,65$ & Treinamento & 0,78 & 0,38 \\
\hline$\Delta \%$ & 0,4 & $-9,31$ & $\begin{array}{l}\text { Grupo } \\
\text { treinamento }\end{array}$ & 0,9 & 0,35 \\
\hline
\end{tabular}

a: grupo experimental; b: grupo controle; c: veia safena parva

\section{DISCUSSÃO}

A insuficiência venosa crônica (IVC) é definida como uma anormalidade da função do sistema venoso causada por uma incompetência valvular associada ou não à obstrução do fluxo sanguíneo ${ }^{(7)}$. Dessa maneira, a IVC pode afetar os dois sistemas venosos (profundo e superficial) de forma conjunta ou isoladamente, e seu surgimento pode resultar tanto a partir de uma desordem congênita como em consequência de hábitos cotidianos que a fazem ser adquirida no decorrer da vida ${ }^{(24)}$.

Observam-se na literatura resultados objetivos quanto aos ganhos de força ${ }^{(1)}$, massa muscular ${ }^{(2)}$, perda de peso e alterações da composição corporal em resposta à prática do treinamento com pesos. No entanto, tais variáveis foram utilizadas apenas para a caracterização da amostra, ou seja, não foram medidas no reteste. Deste modo, não se pode fazer inferências em relação ao comportamento das variáveis após o período de intervenção.

A presente pesquisa ateve-se à avaliação dos diâmetros venosos devido à relação desta variável com as insuficiências do sistema venoso periférico, visto que a dilatação da luz do vaso provoca o refluxo venoso e que, consequentemente, aumenta a pressão venosa local. Tal fato ocorre principalmente nos membros inferiores, lugar em que a coluna de sangue sofre a ação da força gravitacional no seu trajeto ascendente ao átrio direito ${ }^{(14)}$.

Apesar de as medidas dos diâmetros venosos apresentaram elevada variabilidade, as veias demonstraram estar adequadas quanto à sua função e competência, já que após a avaliação com ecodoppler o médico angiologista não referiu a presença de refluxo sanguíneo na amostra investigada. Contudo, salienta-se que esta variável não foi inferida em relação à intervenção adotada. Ressalta-se que o equipamento empregado para as medições é adotado como método padrão para diagnóstico e demonstração da anatomia em casos de doença venosa dos membros inferiores ${ }^{(14,15,19,20)}$.

Com base nos valores de diâmetro venoso em nosso estudo, o programa de exercício com cargas utilizado não promoveu alterações significativas no diâmetro das veias safenas magna e parva em nenhuma de suas porções avaliadas $(p>0,05)$ (tabelas 2,3 e 4). Observa-se que as médias de ambos os grupos foram menores após o treinamento, indicando que a intervenção com pesos não alterou negativamente a estrutura das veias.
Quanto à corroboração ou contestação desses dados com os resultados de outros estudos, coloca-se em evidência que os mecanismos fisiológicos capazes de explicar possíveis alterações ainda não são claros, uma vez que pesquisas não apresentam respostas conclusivas.

Em analogia a outras metodologias, observa-se que os resultados desse estudo estão de acordo com dados apresentados por Van Duijnhoven et al.(25), que, ao aplicarem um programa de treinamento neuromuscular com eletroestimulação, não identificaram aumento no diâmetro da veia poplítea.

Com relação às investigações que objetivaram analisar os efeitos do exercício neuromuscular sobre a competência vascular, os resultados indicam que o fortalecimento do grupo muscular da panturrilha causa benéficas alterações na hemodinâmica venosa, possibilitando a diminuição do refluxo sanguíneo, redução do volume venoso funcional e da fração de volume residual, e aumento da fração de ejeção sanguínea(26).

Lima et al. ${ }^{(7)}$ destacam que a IVC e suas complicações quase sempre estão relacionadas a uma bomba muscular inadequada da panturrilha. Segundo esses autores, a bomba, quando se encontra em perfeito funcionamento, comprime as veias profundas da panturrilha durante a contração muscular, fazendo com que as válvulas distais da veia profunda e das veias perfurantes se fechem e, consequentemente, ejetem o sangue em direção ao coração. Já nos momentos de relaxamento muscular são produzidas grandes quedas de pressão, acarretando a aspiração do sangue em direção às porções distais dos membros inferiores.

A bomba muscular da panturilha, ao exercer essa ação aspirante e compressora, pode reduzir a pressão hidrostática venosa de um indivíduo de $100 \mathrm{mmHg}$ a valores próximos a $0-30 \mathrm{mmHg}$ durante a deambulação $0^{(7)}$, e o exercício físico(5). Ibrahim et al. ${ }^{(27)}$ afirmam que a disfunção dessa bomba é responsável pela hipertensão venosa, a qual desencadeia um excessivo acúmulo de líquido fibrinogênio no tecido subcutâneo, resultando em insuficiência venosa. Acredita-se que as mulheres de nosso estudo foram beneficiadas por esses efeitos; entretanto, para o período de treino proposto, essas modificações podem ter sido limitadas.

Na ocorrência do refluxo venoso, o "coração periférico" tenta compensar a sobrecarga de volume das veias insuficientes promovendo a ejeção de mais sangue ${ }^{(28)}$. O refluxo venoso não foi avaliado no presente estudo e aponta-se como uma das limitações. Porém, a quantificação do diâmetro não deve ser renegada, uma vez que apresenta relação direta com o refluxo observado(16). Engelhorn et al. ${ }^{(15)}$, além de evidenciarem que o diâmetro da veia safena é um fator que exerce potencial interferência na insuficiência valvular venosa e no refluxo, informam que através da análise isolada desta única medida (diâmetro) é possível predizer a existência de refluxo com um nível de acurácia equivalente a $70 \%$.

Em adição, destaca-se que não houve controle sobre informações quanto ao uso regular de medicamentos que possam interferir no funcionamento do sistema venoso dos membros inferiores, e coloca-se a não utilização de um instrumento capaz de mensurar a percepção subjetiva de qualidade de vida da amostra, uma vez que muitas das mulheres participantes relataram a sensação de um elevado bem estar após a realização do programa de exercício. Por fim, ressalta-se que os resultados observados limitam-se às mulheres adultas com graus relativamente baixos de insuficiência venosa crônica (classificação CEAP de um a três).

\section{CONCLUSÃO}

A prática do exercício com cargas pode ser considerada um meio de intervenção ou tratamento, uma vez que não promoveu alterações 
no diâmetro venoso de mulheres que apresentaram insuficiência venosa crônica nos membros inferiores. Os resultados observados limitam-se às mulheres adultas com graus relativamente baixos de insuficiência venosa crônica. Sugere-se a realização de novas investigações em que seja possível avaliar portadores de IVC em seus diversos níveis de gravidade, bem como ser capaz de analisar, de maneira mais precisa, os efeitos que o exercício com cargas exerce sobre outras variáveis consideradas marcadores da (in)competência venosa de membros inferiores.

\section{AGRADECIMENTOS}

Os autores agradecem ao apoio financeiro do CNPq, Programa de Apoio a Planos de Reestruturação e Expansão das Universidades Federais (REUNI) e do Instituto de Apoio a Universidade de Pernambuco (IAUPE).

Todos os autores declararam não haver qualquer potencial conflito de interesses referente a este artigo.

\section{REFERÊNCIAS}

1. Folland JP, Williams AG. The adaptations to strength training: morphological and neurological contributions to increased strength. Sports Med. 2007;37:145-68

2. Abe T, DeHoyos DV, Pollock ML, Garzarella L. Time course for strength and muscle thickness changes following upper and lower body resistance training in men and women. Eur J Appl Physiol. 2000;81:174-80

3. Bocalini DS, Serra AJ, dos Santos L, Murad N, Levy RF. Strength training preserves the bone mineral density of postmenopausal women without hormone replacement therapy. J Aging Health 2009;21:519-27

4. Reeves ND, Maganaris, CN, Narici MV. Effect of strength training on human patella tendon mechanical properties of older individuals. J Physiol. 2003;548:971-81

5. Rowland TW. The circulatory response to exercise: role of the peripheral pump. Int J Sports Med. 2001;22:558-65

6. Beebe-Dimmer JL, Pfeifer JR, Engle JS, Schottenfeld D. The epidemiology of chronic venous insufficiency and varicose veins. Ann Epidemiol. 2005;15:175-84

7. Lima R, Santiago L, Moura R, Souza C, Evangelista S, Gerais Britto R. Efeitos do fortalecimento muscular da panturrilha na hemodinâmica venosa e na qualidade de vida em um portador de insuficiência venosa crônica. J Vasc Bras. 2002;1:219-26.

8. Abenhaim L, Kurz X. The VEINES study (VEnous Insufficiency Epidemiologic and Economic Study): an international cohort study on chronic venous disorders of the leg. VEINES Group. Angiology. 1997:48:59-66.

9. Maffei F. Insuficiência venosa crônica: diagnóstico e tratamento clínico. In Doenças Vasculares Periféricas. F. Maffei, S. Lastoria W. Yoshida. Editors. 1995, MEDSI: Rio de Janeiro.

10. Mashour NH, Lin Gl, Frishman WH. Herbal medicine for the treatment of cardiovascular disease: clinical considerations. Arch Intern Med. 1998;158:2225-34

11. Saharay M, Scurr JH. Minimally invasive surgery for perforator vein incompetence. Cardiovasc Surg. 1996;4:701-5.

12. Blecken SR, Villavicencio JL, Kao TC. Comparison of elastic versus nonelastic compression in bilateral venous ulcers: a randomized trial. J Vasc Surg. 2005;42:1150-5.

13. McDermott MM, Guralnik JM, Ferrucci L, Tian L, Pearce WH, Hoff F, Liu K, Liao Y, Criqui MH. Physical activity, walking exercise, and calf skeletal muscle characteristics in patients with peripheral arterial disease. J Vasc Surg. 2007;46:87-93.

14. Padberg FT, Jr., Johnston, MV, Sisto, SA. Structured exercise improves calf muscle pump function in chronic venous insufficiency: a randomized trial. J Vasc Surg. 2004;39:79-87.
15. Engelhorn C, Engelhorn A, Salles-Cunha S, Picheth F, Castro Jr N, Dabul Jr N, Gomes C. Relationship between reflux and greater saphenous vein diameter. Journal of Vascular Technology. 1997;21:167-71.

16. Mdez-Herrero A, Gutierrez J, Camblor L, Carreno J, Llaneza J, Rguez-Olay J, Suarez E. The relation among the diameter of the great saphenous vein, clinical state and haemodynamic pattern of the saphenofemoral junction in chronic superficial venous insufficiency. Phlebology. 2007;22:207-13.

17. Kistner RL, Eklof B, Masuda EM. Diagnosis of chronic venous disease of the lower extremities: the "CEAP" classification. Mayo Clin Proc. 1996;71:338-45

18. Ferreira M, Matsudo S, Matsudo V, Braggion G. Efeitos de um programa de orientação de atividade física e nutricional sobre o nível de atividade física de mulheres fisicamente ativas de 50 a 72 anos de idade. Rev Bras Med Esporte. 2005;11:172-76.

19. Szendro G, Nicolaides AN, Zukowski AJ, Christopoulos D, Malouf GM, Christodoulou C, Myers K. Duplex scanning in the assessment of deep venous incompetence. J Vasc Surg. 1986;4:237-42.

20. Seidel A, Miranda Jr. F, Juliano Y, Novo, N. Relationship between the diameter of great saphenous vein and body mass index. J Vasc Bras. 2005;4:265-69.

21. Brzycki M. Strength testing - predicting a one-rep max from reps-to-fatigue. (measuring muscular strength). JOPERD. 1993;64:88-90.

22. Whisenant MJ, Panton LB, East WB, Broeder CE. Validation of submaximal prediction equations for the 1 repetition maximum bench press test on a group of collegiate football players. J Strength Cond Res. 2003;17:221-7.

23. ACSM. American College of Sports Medicine position stand. Progression models in resistance training for healthy adults. Med Sci Sports Exerc. 2009;41:687-708

24. Porter JM, Moneta GL. Reporting standards in venous disease: an update. International Consensus Committee on Chronic Venous Disease. J Vasc Surg. 1995;21:635-45.

25. Van Duijnhoven NT, Bleeker MW, de Groot PC, Thijssen DH, Felsenberg D, Rittweger J, Hopman MT. The effect of bed rest and an exercise countermeasure on leg venous function. Eur J Appl Physiol. 2008;104:991-8.

26. Orsted HL, Radke L, Gorst R. The impact of musculoskeletal changes on the dynamics of the calf muscle pump. Ostomy Wound Manage. 2001;47:18-24.

27. Ibrahim S, MacPherson DR, Goldhaber, SZ. Chronic venous insufficiency: mechanisms and management Am Heart J. 1996;132:856-60.

28. Simka M. Calf muscle pump impairment and delayed healing of venous leg ulcers: air plethysmographic findings. J Dermatol. 2007;34:537-44 


\section{ERRATA}

Na RBME, volume 16, n 6, novembro/dezembro de 2010, no artigo "Treinamento de sobrecarga muscular não afeta o diâmetro das principais veias dos membros inferiores em mulheres adultas com insuficiência venosa", as tabelas 3 e 4, nas páginas 415 e 416, respectivamente, devem ser substituídas pelas respectivas tabelas 3 e 4 a seguir:

Tabela 3. Diâmetros da veia safena magna em nível da coxa nos momentos pré e pós-treinamento.

\begin{tabular}{|c|c|c|c|c|c|}
\hline Variável/teste & $\mathrm{GE}^{\mathrm{a}}(\mathrm{n}=10)$ & $\mathrm{GC}^{\mathrm{b}}(\mathrm{n}=10)$ & Efeitos & $F$ & $P$ \\
\hline $\begin{array}{c}\text { VSMCc } \\
\text { superior } \\
\end{array}$ & & & ANOVA & & \\
\hline $\begin{array}{c}\text { Pré- } \\
\text { treinamento }\end{array}$ & $4,08 \pm 1,55$ & $3,69 \pm 0,90$ & Grupo & 0,47 & 0,49 \\
\hline $\begin{array}{c}\text { Pós- } \\
\text { treinamento }\end{array}$ & $3,90 \pm 1,68$ & $3,53 \pm 1,28$ & Treinamento & 0,75 & 0,39 \\
\hline$\Delta \%$ & $-4,61$ & $-4,53$ & Grupo* Treinamento & 0,00 & 0,97 \\
\hline VSMC médio & & & ANOVA & & \\
\hline $\begin{array}{c}\text { Pré- } \\
\text { treinamento }\end{array}$ & $3,13 \pm 0,95$ & $3,51 \pm 1,03$ & Grupo & 0,31 & 0,57 \\
\hline $\begin{array}{c}\text { Pós- } \\
\text { treinamento }\end{array}$ & $3,05 \pm 1,05$ & $3,15 \pm 1,14$ & Treinamento & 2,24 & 0,14 \\
\hline$\Delta \%$ & $-2,62$ & $-11,42$ & Grupo* Treinamento & 0,87 & 0,36 \\
\hline VSMC Inferior & & & ANOVA & & \\
\hline $\begin{array}{c}\text { Pré- } \\
\text { treinamento }\end{array}$ & $3,13 \pm 1,00$ & $3,37 \pm 0,93$ & Grupo & 0,42 & 0,52 \\
\hline $\begin{array}{c}\text { Pós- } \\
\text { treinamento }\end{array}$ & $2,96 \pm 1,11$ & $3,28 \pm 1,23$ & Treinamento & 0,50 & 0,48 \\
\hline$\Delta \%$ & $-5,74$ & $-2,74$ & Grupo* Treinamento & 0,45 & 0,83 \\
\hline
\end{tabular}

Tabela 4. Diâmetros da veia safena parva nos momentos pré e póstreinamento.

\begin{tabular}{c|c|c|c|c|c}
\hline Variável/teste & $\mathbf{G E}^{\mathbf{a}}(\mathbf{n}=\mathbf{1 0})$ & $\mathbf{G C}^{\mathbf{b}} \mathbf{( n = 1 0 )}$ & Efeitos & $\mathbf{F}$ & $\mathbf{P}$ \\
\hline VSPc superior & & & ANOVA & & \\
\hline Pré-treinamento & $2,55 \pm 0,71$ & $2,45 \pm 0,62$ & Grupo & 0,23 & 0,63 \\
\hline Pós-treinamento & $2,59 \pm 0,97$ & $2,39 \pm 0,65$ & Treinamento & 0,00 & 0,93 \\
\hline$\Delta \%$ & $+1,54$ & $-2,51$ & Grupo* Treinamento & 0,22 & 0,63 \\
\hline VSP Médio & & & ANOVA & & \\
\hline Pré-treinamento & $2,46 \pm 0,55$ & $2,51 \pm 0,53$ & Grupo & 0,11 & 0,74 \\
\hline Pós-treinamento & $2,30 \pm 0,55$ & $2,40 \pm 0,55$ & Treinamento & 1,78 & 0,19 \\
\hline$\Delta \%$ & $-6,95$ & $-4,58$ & Grupo* Treinamento & 0,07 & 0,78 \\
\hline VSP Inferior & & & ANOVA & & \\
\hline Pré-treinamento & $2,25 \pm 0,38$ & $2,23 \pm 0,54$ & Grupo & 0,00 & 0,96 \\
\hline Pós-treinamento & $2,08 \pm 0,57$ & $2,13 \pm 0,59$ & Treinamento & 1,65 & 0,21 \\
\hline \multicolumn{7}{|c|}{$\Delta \%$} & $-8,17$ & $-4,69$ & Grupo* Treinamento & 0,12 & 0,72 \\
\hline a: grupo experimental; b: grupo controle; c: veia safena parva.
\end{tabular}

